\title{
Topological Assessment of Unidentified Moving Objects
}

\author{
Arturo Tozzi \\ Center for Nonlinear Science, Department of Physics, University of North Texas, Denton, Texas, USA \\ 1155 Union Circle, \#311427Denton, TX 76203-5017 USA \\ tozziarturo@libero.it \\ Arturo.Tozzi@unt.edu
}

James F. Peters

Department of Electrical \& Computer Engineering, University of Manitoba, Winnpeg R3T 5V6 Manitoba Canada and Department of Mathematics, Adıyaman University, 02040 Adıyaman, Turkey

James.peters3@umanitoba.ca

Starting from unidentified objects moving inside a two-dimensional Euclidean manifold, we propose a simple method to detect the topological changes that occur during their reciprocal interactions and shape morphing. This method, which allows the detection of topological holes development and disappearance, makes it possible to solve the uncertainty due to disconnectedness, lack of information and absence of objects' sharp boundaries, i.e., the three troubling issues which prevent scientists to select the required proper sets/subsets during their experimental assessment of natural and artificial dynamical phenomena, such as fire propagation, wireless sensor networks, migration flows, neural networks' and cosmic bodies' analysis.

KEYWORDS: holes; grid; shapes; homology; uncertainty.

The tremendous power of set and cell complex theory (Peters, 2018a, 2018b; Cooke, Finney, 1967; Alexandroff, 1935) has been successfully used in many applications, from math to logic, from physics to biology and statistics. In a covering of a finite, bounded plane region, for example, there are three elementary cells to consider, namely, 0 -cells (vertexes), 1cells (edges) and 2-cells (filled triangles). The core of set and cell complex theory lies in the occurrence of path-connected sets or subsets, i.e., the presence of elements which are (more or less arbitrarily) joined together and separated from the external environment. A serious problem arises when we grasp that, in many applications, a system's object shapes are unknown and apparently disconnected, making it very difficult to find and recognize the proper subtending sets' very presence and/or dynamics. Indeed, the boundaries of object shapes may be very irregular and indistinct, leading to a knowledge of the split between shape interiors and boundaries which hampers shape approximation and measurement. For example, cause/effect relationships could be inescapably hidden from our observation, if we are unable to pin down and accurately measure systems' components and/or events. In this paper, we will start from moving objects, i.e., features which can be described by a function from time to its corresponding spatial object (Gäuting et al., 2000). Similarly, a moving region related to a moving object displays mappings from time to a region object, i.e., a feature consisting of one or several connected components called faces, each of which may or may not have holes inside it (Schneider and Behr, 2006).

Several approaches to the study of moving and region objects use procedures of data analysis arising from persistent homology, whose early roots (for connected components) trace back to Size Theory (Verri et al., 1993; Verri and Uras, 1997), later generalized to higher dimensions (Edelsbrunnerer al., 2002; Edelsbrunner and Morozov, 2012). Among the models which assess topological relationships, i.e., the topological properties of a given spatial relationship (Corcoran and Jones, 2018), the most successful are the Intersection Model (Egenhofer, 1991) and the Region Connection Calculus (Randell et al., 1992). Still, both these approaches and their generalizations (Schneider and Behr, 2006) assume that the locations of connected objects, modelled as subsets of $\mathrm{R}^{2}$, are accurately known. This is not always the case, because confounding factors may cause uncertainty in spatial data detection: among them, the most significant are incompleteness, due to lack of information, and vagueness, resulting from objects not having crisp or sharp boundaries (Worboys, 1998). In this paper, in order to tackle the painstaking issues related to unavailable topological spatial relationships and intrinsic uncertainty, we tackle the goal to assess the trajectories of undefined/disjointed/disconnected sets or subsets: in particular, we consider changes in topological features of two unknown spatial shapes moving inside a two-dimensional grid. We aim to evaluate spatial objects whose geometries and shapes change over time: we will term these objects with the abovementioned definition of moving regions (Liu and Schneider, 2011) and will propose a model able to detect their conceivable topological modifications. 


\section{MATERIALS AND METHODS}

In technical terms, our goal was to achieve topological features to describe intersection or union of spatial moving disconnected regions in a two-dimensional abelian lattice. At first, we built a two-dimensional grid made by $10 x 10$ squares, each standing for a single pixel. The grid is composed of 100 white squares, representing empty locations. We randomly generated a pair of two-dimensional objects with different shapes, free to move inside the grid with unpredictable trajectories. The presence and movements of objects inside the grid are illustrated by pixels turning red from white with time passing. The two different objects, which may display changing shapes, were allowed to superimpose and perform intersection and union operations. We benefited from the fact that a moving region whose location and extend change over time can imply topological changes such as and hole formation and disappearance.

Hole detection was based on snapshots captured at instants termed observations. We formally defined a basic topological change, i.e., hole formation, between two consecutive observations. The detection method, modified from Gäuting et al. (2000), uniquely maps a unit, by partitioning the observations before and after the changes. A moving region can have different holes at different time intervals, and different changes between two same states may occur. For example, from a simple region to a single region with holes, either a hole is formed inside the region (termed hole form), or the region touches itself at two ends (termed region self-touch).

The grid was equipped with sensors able to detect just a single feature: an empty square (a white pixel) fully surrounded by eight filled squares (red pixels). In other words, the sensors, unable to detect object's shapes, occurrence and movements, are able to spot just the dynamical presence and disappearance of holes in the grid. Here follows the sensors' detection algorithm. At the Earth's near-ground level, holes can be found in surface object punctures and atmospheric water vapor puncture. From a video camera perspective, holes are light-absorbing voids (Peters 2019). Both types of holes vary minutely in size and extent over time. Atmospheric holes are continuously varying in location due to changing atmospheric pressure plasmas (Overzet et al., 2010). Variations in atmospheric holes also vary due to movements of water molecules in the air and shifting sunlight conditions. Evidence of this can be seen in the sequence of video frames in the Figure below:
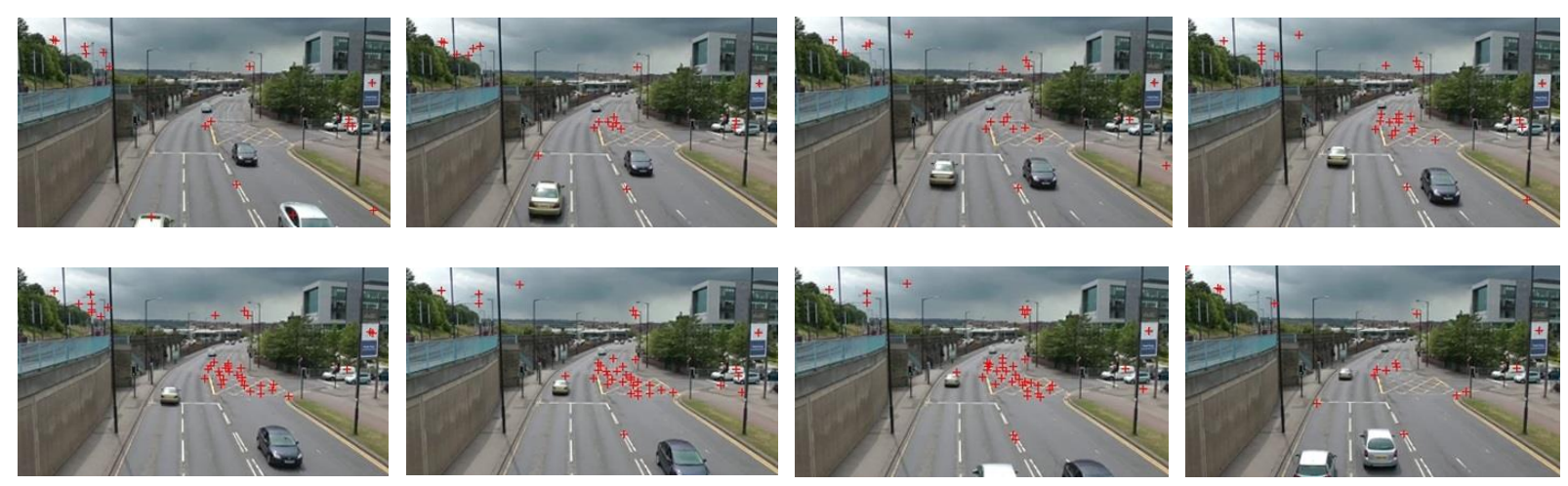

Figure. Centroids on holes in a sequence of video frames. Each + identifies the centroid of a hole. The locations of the holes in these video frames vary from moment due varying atmospheric conditions. 
Sample centroids (holes) of 2D and 3D regions are shown here:

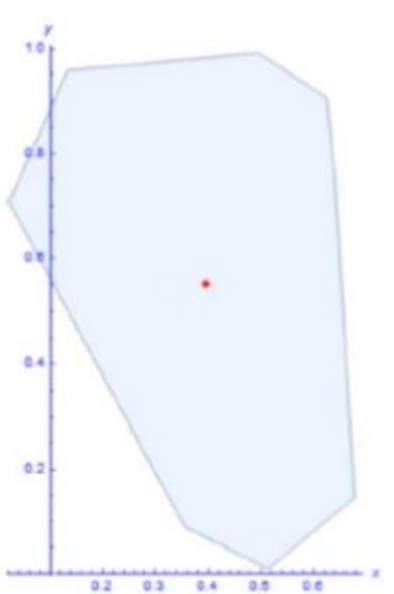

A. Centroid at $(\mathbf{x}, \mathrm{y})=(\mathbf{0 . 3 9 6}, 0.554)$

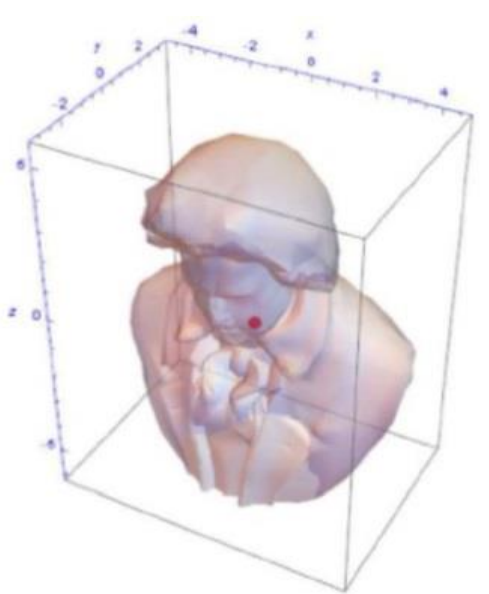

B. Centroid at $(x, y, z)=(0.0005,0.0504,-0.7149)$

The centroid of a region is the center of mass of the region. Let $\left(x_{i}, y_{j}\right), i=1, \ldots, n, j=1, \ldots, m$ be the coordinates of the points in an $n \times m$ region. And let $\left(x_{c}, y_{c}\right)$ be the coordinates of the region centroid. Then the centroid of this $2 \mathrm{D}$ region is located at $\left(x_{c}, y_{c}\right)=\left(\frac{1}{n} \sum_{i=1}^{n} x_{i}, \frac{1}{m} \sum_{j=1}^{m} y_{i}\right)$.

Similarly, a centroid in a 3D region such as the regions in the video frames in the Figure above is located at $\left(x_{c}, y_{c}, z_{c}\right)=\left(\frac{1}{n} \sum_{i=1}^{n} x_{i}, \frac{1}{m} \sum_{j=1}^{m} y_{i}, \frac{1}{o} \sum_{k=1}^{o} y_{k}\right)$.

The algorithm used to find the centroids of holes found in the video frames in the Figure above is given next. 
Algorithm Centroids of Video Frame Holes. Input: Colour image img in a video frame. Output: Centroids on holes recorded in a video frame.

Img $\rightarrow$ imgGrey \% Convert img to greyscale.
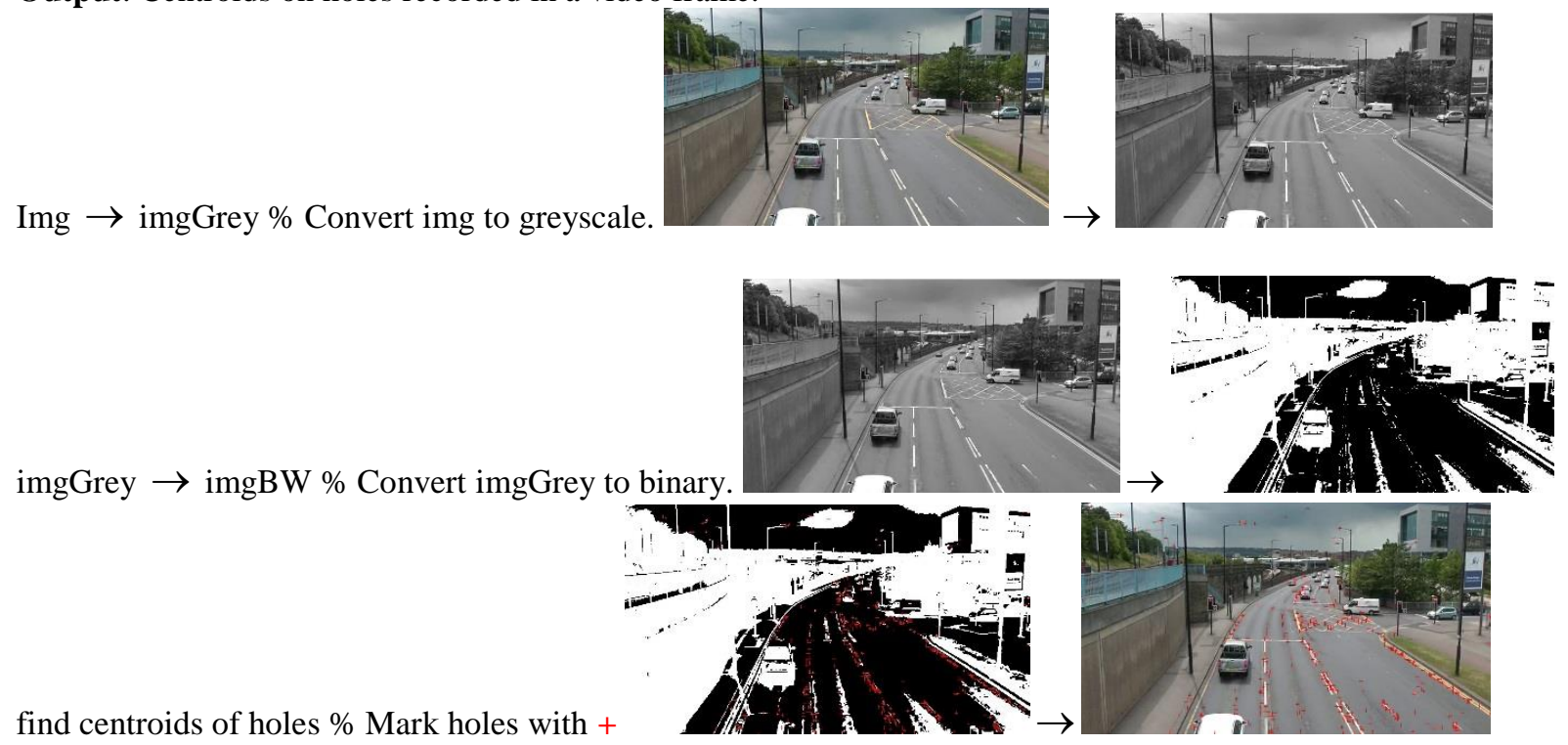

End Algorithm Centroids of Video Frame Holes.

\section{RESULTS}

Our technique allows the detection of holes in consecutive images of moving regions traveling in the 10x10 grid.

The Figure illustrates three on the countless possible combinations. At first, we assess the case of two intersecting moving figures (Figure C). Also, we consider the case which can be explained as region merge, followed by hole form, or vice versa (Figure B). Our account can be generalized to the random interactions of two moving regions equipped with different number of holes (Figure C). It is noteworthy that the knowledge of a sole parameter, i.e., the firing pixels which stand for the holes arising from the dynamical interaction of the two moving regions, allows us to achieve knowledge about topological features of otherwise undetectable objects. Indeed, even if an observer was unaware of the two moving objects' shapes and trajectories, he would be still able to extract useful information, simply looking at the detection screening that signals the presence (and disappearance) of holes. Although no information is available concerning the two objects' shape, nevertheless the observer is still able to detect and quantify their dynamics and interactions. 
Time 0

A

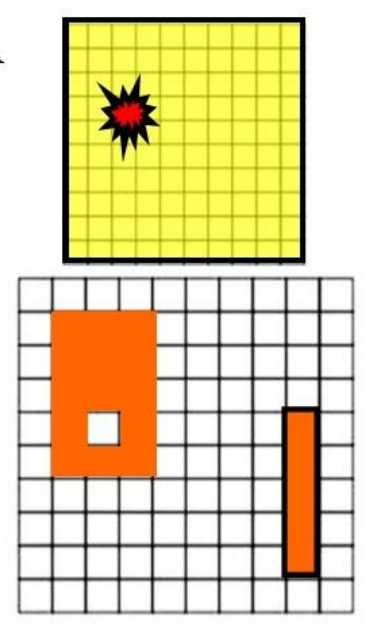

B
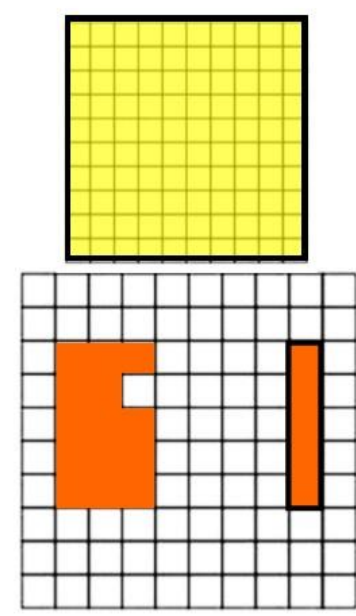

C
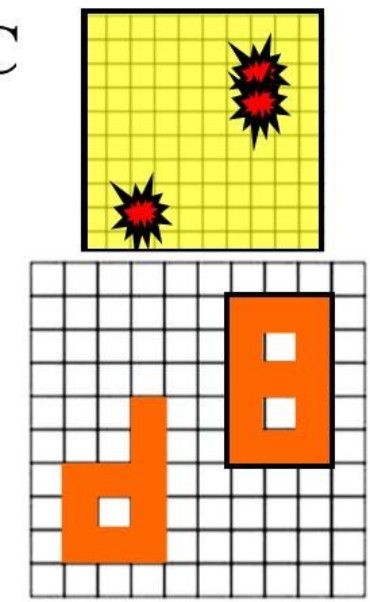

Time 1
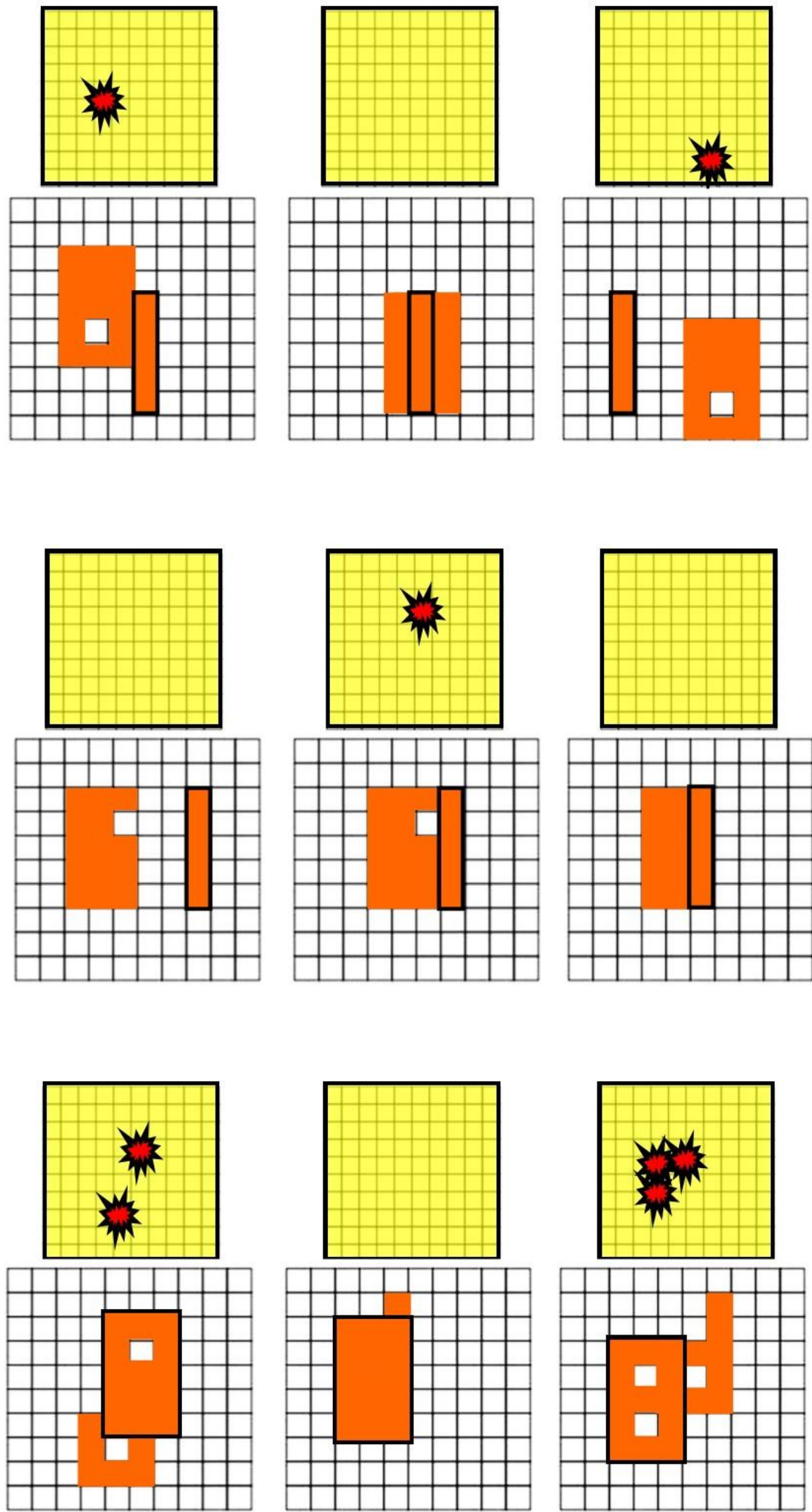

Time 2
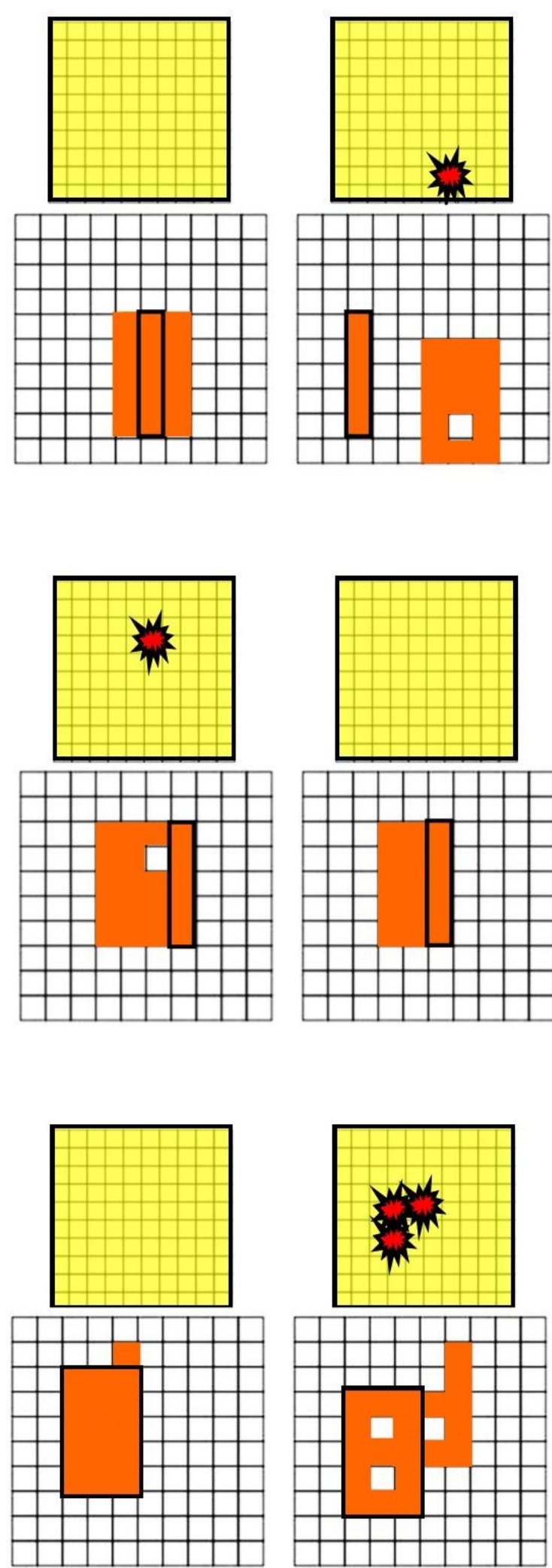

Time 3

更


stars). The lower part of the three figures (white grids encompassing red shapes) illustrates moving regions traveling inside a 10x10 grid. Figure A depicts the union of two objects, one with a hole and one without. Figure B displays a case of hole formation, when two shapes with ho holes interact and merge. Figure $\mathbf{C}$ depicts the dynamics of two moving regions equipped with a different number of holes.

\section{CONCLUSIONS}

Demarcation among objects and things is somewhat arbitrary, because our mind tends to exclude the continuity among hidden or unknown structures of the world (Fort, 1919). We are used to draw lines of separation among things that we judge different, arbitrarily excluding or including issues in our description, to achieve positive demarcations that allow a pragmatic treatment of the world based on regularity and uniformity (Popkin and Maia Neto, 2007; Autrecourt, 1340). In touch with set theory, observers tend to spatially and temporally split the set of the entire world in different, arbitrary, fictious subsets that could not really stand for different objects or events. The same scientific concept of "observable" lies on the choice of variables in peculiar experimental settings: scientists, putting aside most of the variables, focus their efforts on a few features. For example, elementary particles are fully defined just in terms of three experimental observables, i.e., charge, spin and mass, letting aside, for practical purposes, less "useful" features. There's a plenty of boundaries in math, physics and biology. In these disciplines, the concept of boundary is grounded on the presence of internal and external surfaces. This concept seems straightforward in math, and in particular in topology. In algebraic topology, a Jordan curve is a plane simple closed curve, i.e., a non-self-intersecting continuous loop in the plane surfaces (Ghrist 2014). This gives rise to the (apparently) obvious Jordan curve theorem, which says that every Jordan curve divides the plane into an "interior" region bounded by the curve and an "exterior" region containing all of the nearby and far away exterior points. Therefore, in topology, despite the analytic description of disconnected sets has been extensively provided (Arkhangelskii 2001; Terasawa, 2004), we are in front of a rather simple statement: there exist an "inside" and an "outside" region.

Concerning the biophysical realm, many accounts of our real world, both philosophical and biological, are used to think at a living being as an individual, self-preserving, unique subject equipped with borders, such as cellular membranes, envelopments, Markov blankets, and so on. The canonical illustration of biological organisms depicts "something" equipped with some peculiar activity, confined into itself, which struggles against (and cooperates with) the external world, in order to keep its entropy as low as possible and devoted to self-preservation (Ramstead et al., 2019). However, despite such stereotypical descriptions, the things get much more complicated when we approach the real word and its physical/biological content. The demarcation among objects, things and living beings could be somewhat arbitrary, and the same holds for set theory too. The same limitation holds for the very concept of evolution, which is based on species. Our concept of species is very arbitrary, because it throws not experimentally demonstrated borders among living beings. If we think to different populations of Homo. despite we are used to consider Sapiens, Neandertals, Denisovians, Floresians as different species, genetic studies undoubtedly point towards their ancient ibridation, which occurred more than once in different prehistorical contexts (Slon et al., 2018). Concerning our DNA, the delimitation of a species from another is sometimes difficult, due, e.g., to the presence in animal genome of viral and bacterial sequences. To make an example, the horizontal transmission of genetic material is able to overtake the so-called "species-specific" barriers (Chen et al., 2018), making sometimes difficult to clearly encompass individual living beings in a given species.

Given these premises, our aim here was to describe a technique to detect topological information from unknown and (possibly) disconnected spatial dynamic features. We showed how unidentified interactions between two shapes on a two-dimensional grid give rise to modifications in topological features which can be detected and quantified. This means that topological changes pave the way to several possible applications in far-flung scientific disciplines. To provide a few examples, the detection of moving regions' holes might allow to assess the extent of a spreading forest fire, or the coverage of two mobile networking devices (Liu and Schneider, 2011). The study of these topological changes might too help researchers to improve the performance of wireless sensor networks (Worboys and Duckham, 2006) and the knowledge of protein folding dynamics (Tozzi et al., 2016). Also, topological detection in network models might provide insights in both global structure and local node interactions, either in natural or artificial neural networks and in lattice percolation procedures. Further, unknown patterns assessed through topological weapons could be useful in understanding economic and sociologic trends of migratory fluxes (Ignacio and Darcy, 2019). Our technique of hole detection might also allow to assess the discrete dynamic changes and multi-dynamic clustering occurring at the macroscales of galaxies, cosmic bodies and Megaparsec web-like cosmic matter distribution, describing the topological invariants of their subtending networks (Pranav et al., 2016) and, possibly, providing better insights in dark matter and vacuum energy. Our account might also provide unexpected insights in the physiological neural mechanisms of figureground perception, in scenes where near surfaces occlude multiple contours and borders at different depths, producing alignments that are improbable except under conditions of occlusion. Indeed, in touch with our account, Gillam and Grove (2011) hypothesized that figure-ground and hole perception can be determined solely by properties of ground, in the absence of any figural shape, or surround, factors. The last, but not the least, the foremost role of holes seems to corroborate the recently-introduced axiomatic foundations of the descriptive closeness of points and sets, which aims to 
overtake the conventional closeness of points and sets (DiConcilio, et al.,2018). In the context of descriptive proximity, the closeness of points and sets can be viewed in terms of their overlapping descriptions which produce detectable holes.

\section{REFERENCES}

1) Alexandroff P. 1961. Elementary Concepts of Topology. Dover Pubs., Inc., NY.

2) Autrecourt, Nicholas of. About 1340. The Universal Treatise. Marquette University Press, Milwaukee, Wisconsin, 1971.

3) Arkhangelskii AV (2001) [1994]. Extremally-disconnected space, in Hazewinkel, Michiel, Encyclopedia of Mathematics, Springer Science+Business Media B.V. / Kluwer Academic Publishers, ISBN 978-1-55608-0104.

4) Chen J, Quiles-Puchalt N, Chiang YN, Bacigalupe R, Fillol-Salom A, et al. 2018. Genome hypermobility by lateral transduction. Science, Vol. 362, Issue 6411, pp. 207-212. DOI: 10.1126/science.aat5867.

5) Cooke GE, Finney RL. 1967. Homology of Cell Complexes. Princeton University Press, Princeton, NJ.

6) Corcoran P, Jones CB. 2018. Stability and Statistical Inferences in the Space of Topological Spatial Relationships. IEEE Access, 6: 18907 - 18919. DOI: 10.1109/ACCESS.2018.2817493.

7) Di Concilio A., Guadagni C., Peters JF, Ramanna S. 2018. Descriptive proximities. Properties and interplay between classical proximities and overlap. Mathematics in Computer Science 12, 91-106, DOI 10.1007/s11786017-0328-y.

8) Edelsbrunner H, Letscher D, Zomorodian A. 2002. Topological persistence and simplification. Discrete Comput Geom. https://doi.org/10.1007/s00454-002-2885-2

9) Edelsbrunner H, Morozov D (2012) Persistent homology: theory and practice. European Congress of Mathematics, Krakow, 2-7 July, 2012, Europ Math Soc, 31-50

10) Egenhofer MJ. 1991. Reasoning about binary topological relations. Symposium on Spatial Databases. Springer, 141-160.

11) Fort C. 1919. The bbok of damned. Prometheus Books, 1991.

12) Gäuting RH, Bäohlen MH, Erwig M, Lorentzos CSJNA, Schneider M, Vazirgiannis M. 2000. A Foundation for Representing and Querying Moving Objects. ACM Trans. on Database Systems (TODS), 25(1):142, 2000.

13) Ghrist R. 2014. Elementary Applied Topology, Amazon, ISBN: 978-1-5028-8085-1.

14) Gillam BJ, Grove PM. 2011. Contour Entropy: A New Determinant of Perceiving Ground or a Hole. Journal of Experimental Psychology. Human Perception and Performance, 37(3): 750-757.

15) Overzet LJ, Jung D, Mandra MA, Goeckner M., Dufour T, Dussart R, Lefaucheux P. 2010. The European Physical Journal D 60, 449-454, DOI: 10.1140/epjd/e2010-00274-5

16) Ignacio PSP, Darcy IK. 2019. Tracing patterns and shapes in remittance and migration networks via persistent homology. EPJ Data Sci. (2019) 8: 1. https://doi.org/10.1140/epjds/s13688-018-0179-z.

17) Liu H, Schneider M. 2011. Tracking continuous topological changes of complex moving regions. Proceeding SAC '11. Proceedings of the 2011 ACM Symposium on Applied Computing. Pages 833-838. TaiChung, Taiwan. ACM New York, NY, USA. ISBN: 978-1-4503-0113-8 doi>10.1145/1982185.1982366.

18) Peters JF. 2018a. Proximal planar shapes. Correspondence between triangulated shapes and nerve complexes. Bull. of the Allahabad Mathematical Society 33, 113-137. MR3793556, Zbl 06937935

19) Peters, JF. 2018b. Proximal vortex cycles and vortex nerve structures. non-concentric, nesting, possibly overlapping homology cell complexes. Journal of Mathematical Sciences and Modelling 1(2), 56-72.

20) Peters JF. 2019. Computational Geometry, Topology and Physics of Digital Images. Shape Complexes, Optical Vortex Nerves and Proximities. Springer Nature, Switzerland, to appear.

21) Popkin RH, Maia Neto JR (Editors). 2007. Skepticism: An Anthology. Prometheus Books. ISBN 1591024749 (ISBN13: 9781591024743).

22) Pranav P, Edelsbrunner H, van de Weygaert R Vegter G, Kerber M, et al. 2016. The topology of the cosmic web in terms of persistent Betti numbers. Monthly Notices of the Royal Astronomical Society, Volume 465, Issue 4, 11 March 2017, Pages 4281-4310, https://doi.org/10.1093/mnras/stw2862.

23) Ramstead MJD, Constant A, Badcock PB, Friston KJ. 2019. Variational ecology and the physics of sentient systems. Physics of Life Reviews. In press. https://doi.org/10.1016/j.plrev.2018.12.002.

24) Randell DA, Cui Z, Cohn AG. 1992. A spatial logic based on regions and connection. in Proceedings 3rd international conference on knowledge representation and reasoning, 1992. 
25) Schneider M, Behr T. 2006. Topological relationships between complex spatial objects. ACM Transactions on Database Systems (TODS), vol. 31, no. 1, pp. 39-81, 2006.

26) Slon V, Mafessoni F, Vernot B, de Filippo C, Grote S, et al. 2018. The genome of the offspring of a Neanderthal mother and a Denisovan father. Nature, 561, 113-116.

27) Terasawa J. 2004. Extremally Disconnected Spaces. In book: Encyclopedia of General TopologyChapter: G. Special SpacesPublisher: ElsevierEditors: K.P.Hart, Jun-iti Nagata, Jerry E.Vaughan. DOI: 10.1016/B978044450355-8/50092-1.

28) Tozzi A, Fla Tor, Peters JF. 2016. Building a minimum frustration framework for brain functions in long timescales. J Neurosci Res.94(8): 702-716.

29) Verri A, Uras C, Frosini P, Ferri M. 1993. On the use of size functions for shape analysis. Biol Cybern 70:99107.

30) Verri A, Uras C. 1997. Metric-topological approach to shape representation and recognition. Image Vis Comput 14:189-207

31) Worboys M. 1998. Imprecision in finite resolution spatial data. GeoInformatica, vol. 2, no. 3, pp. 257-279, 1998.

32) Worboys M, Duckham M. 2006. Monitoring qualitative spatiotemporal change for geosensor networks. International Journal of Geographical Information Science, 20:1087\{1108, 2006. 